\title{
A Vehicle Speed Measurement System for Nighttime with Camera
}

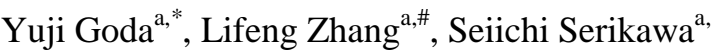 \\ ${ }^{a}$ Electrical Engineering and Electronics, Kyushu Institute of Technology \\ 1-1 sensui-cho, Tobata-ku, Kitakyushu city, Fukuoka 804-8550, Japan \\ "m108303y@tobata.isc.kyutech.ac.jp \\ \#zhang@elcs.kyutech.ac.jp
}

\begin{abstract}
This research proposes a new vehicle speed measuring approach based on image processing technique. This approach is aimed to improve the vehicle speed measuring accuracy at night. Ordinarily, it is difficult to take a clear image at night by using a low profile digital camera. Thus the image based vehicle speed measuring system for dark environment was not developed by now. In this research, the disadvantage of low profile camera is used for speed measuring. By controlling the shutter speed of camera, a bright line comes from the headlamp of a moving vehicle is appeared in the picture. In this work, an algorithm depend on this feature is developed. Experiment result shows a reasonable measuring value.
\end{abstract}

Keywords: Image, processing, Low profile camera, vehicle speed measurement, shutter speed, line of light.

\section{Introduction}

As the amount of traffic increases, the car accidents due to the over speed are occurring frequently. The annual report distributed by Police Traffic Bureau of Japan shows that the $36.2 \%$ of fatal traffic accident comes from the over speed[1]. Therefore, it is very important task to make an effort to prevent the over speed car accident occurrence. In the past 30 years, an automatic speed violation control device called ORBIS has been applied. Such a kind of system using loop coil or radar needs a high deployment costs and maintenance cost, and has a drawback that misdetection occurs in some certain situations. Furthermore, many aged devices are fault and left without repairing[2].

In this situation, recently, image processing based vehicle speed measuring techniques are developed actively[7]. Image processing approach can gives a picture record logs, and can helps the analysis of the cause of the accident efficiently. But image processing based speed

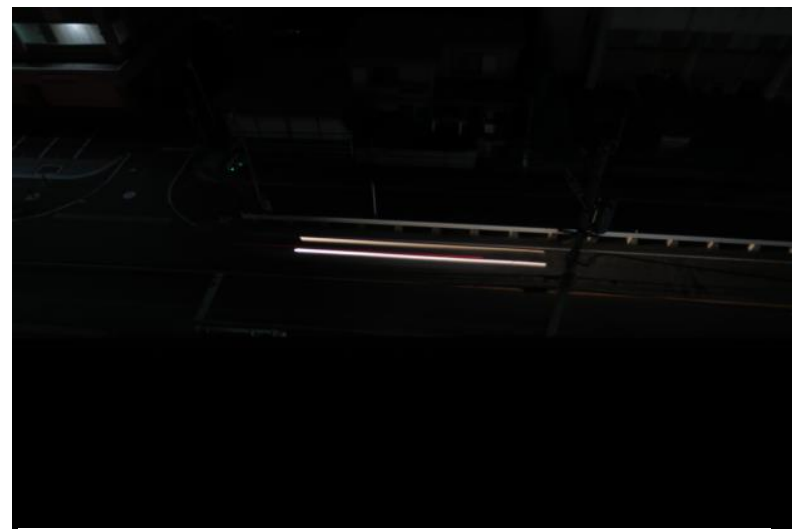

Fig. 1. Image of vehicle running at night.

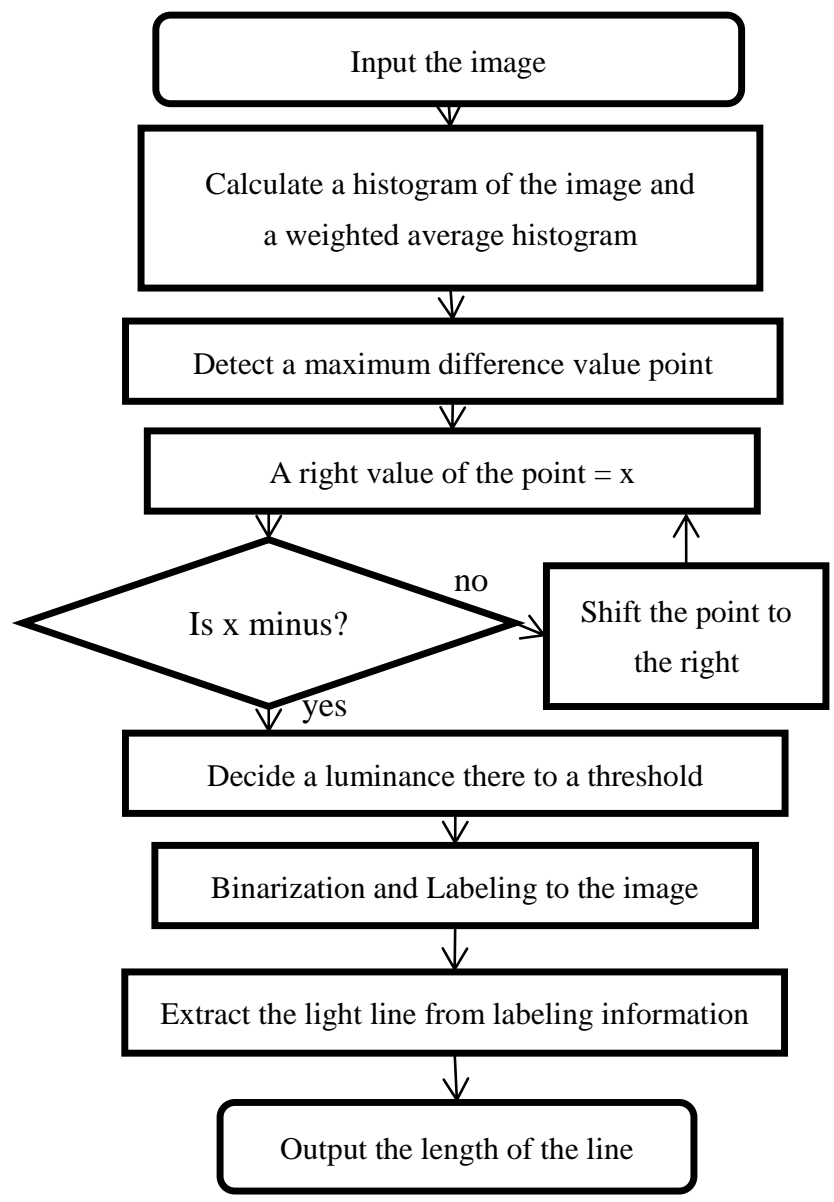

Fig. 1. Flowchart of the light line extraction. 
measuring system also have problems. Especially for a dark circumstance, the pictures cannot be taken clearly and the measuring accuracy becomes low.

In this research, a very new idea is attempted to speed measuring task in the night environment. What we need is a low profile camera, a microcomputer board, and some camera depend parameters like focus depth, imaging area size, etc. Such a construction can yield a low cost system so that it can be applied worldwide. Especially for the newly developing countries, which show a significant increasing rate of vehicle.

The main purpose of this research is to improve accuracy of measuring car speed in the night environment. But in a dark circumstance, it is impossible to take a clear and a sharp picture by using a high shutter speed with a low profile camera. Therefore, we take an opposite operation, take a picture of the moving vehicle first by the setting the camera's shutter speed to 1 second. Thereby, because the camera is fixed and the vehicle is moving, the headlight of vehicle observed in the picture becomes a light line as showed in Fig. 1. Next, the positions of the light lines are detected by using an automatic threshold decision algorithm, and then the line are extracted from a picture by using a labelling method. After that the length of the light line is detected. The vehicle speed is calculated from this length finally.

\section{Theory and Method}

This section explains how to extract the line of light and how to calculate the real distance from the light line length derived from the image.

\subsection{Length of the light line}

The flowchart of the light line extraction is shown Fig. 2. Fig. 1 is an experimental example taken in a real night circumstance. The following processes of proposed method are performed on this picture. In this study, we developed an automatic threshold decision method by using a histogram.

(a) Calculate a histogram of the image and its weighted average histogram

A histogram is calculated from the picture. The picture of result is Fig. 3. Fig .3 is the red-green-blue (RGB) histogram, and the vertical axis is for the number of pixels, and the horizontal for illumination ranging from 0 to 255 . The left histogram in Fig. 3 is blue, the center is green, and the light is red. With the original histogram in Fig. 3 whose

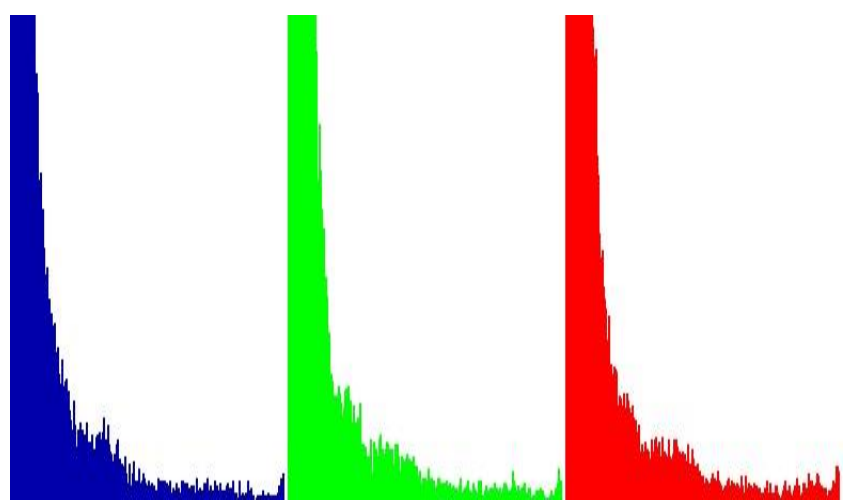

Fig. 3. Histogram of Fig. 1.

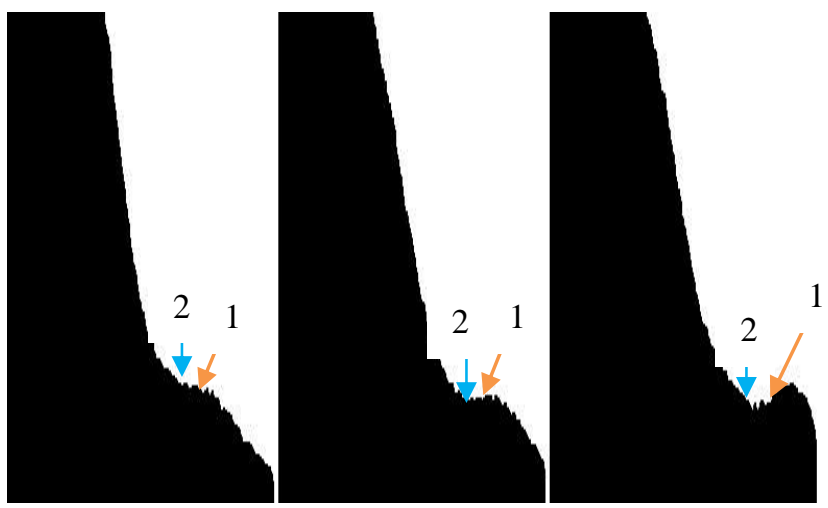

Fig. 4. Histogram of weighted average.

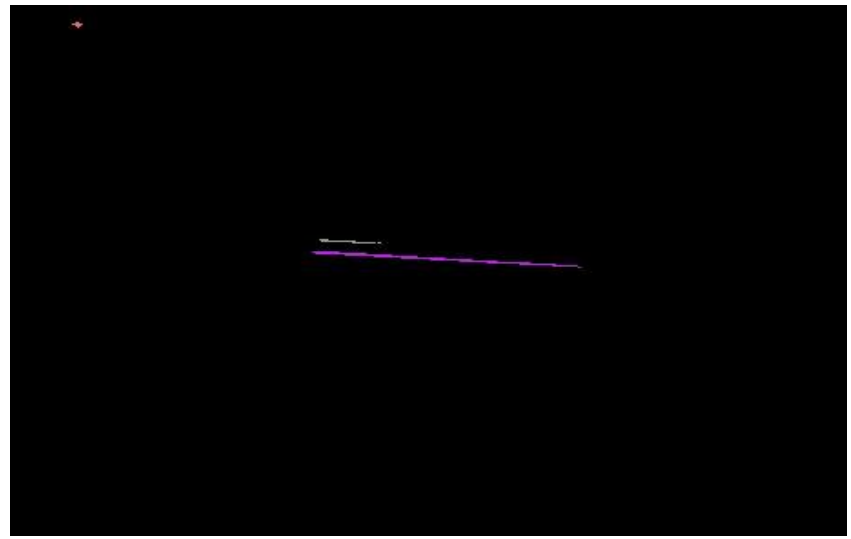

Fig. 5. Threshold-processed image of Fig. 1.

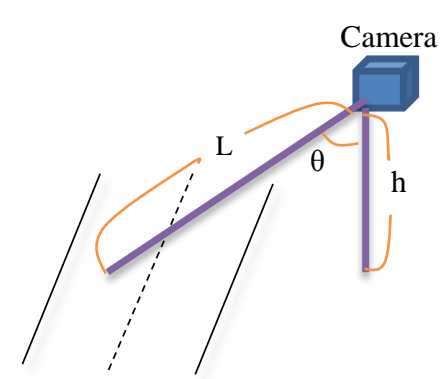

Image

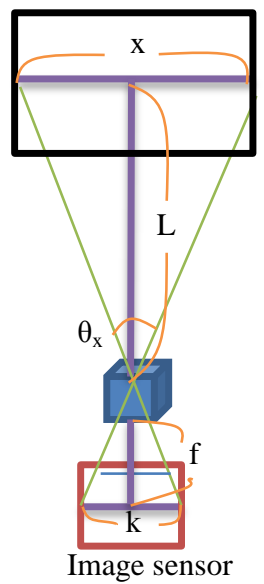

Fig. 6. Camera setting parameters. 
values are changing violently in local area like noise, it is difficult to decide a threshold value. To solve this problem, a Gaussian distributed weight function is utilized to get a smoothed histogram. Fig. 4 shows the result of Gaussian weighted histogram of Fig. 3. Similarly the vertical axis is for the number of pixels, and the horizontal for illumination ranging from 0 to 255. The left histogram in Fig. 4 is blue, the center is green, and the light is red.

(b) Maximum difference value point detection

Applying a differential processing to the weighted average histogram, we can get the highest difference value. This means the maximum slope of the histogram is found, and we use this slope value and its position for threshold value decision. In Fig. 4, the positions pointed by arrow "1" are maxima derivatives of $\mathrm{R}, \mathrm{G}$, and $\mathrm{B}$ color channels respectively.

(c) Threshold decision

As we known, the light line has a higher brightness value than other area of the picture in the dark environment like the night. Therefore, we inspect the Gaussian weighted histogram from right side to the left side, and finding the first minus difference value position at the left side of the found maximum difference value in previous step. Then, we decide the luminance value there as a threshold value. The luminance values pointed by arrow "2" shown in Fig. 4 are decided as threshold values of each color channel.

\section{(d) Binarization and labeling}

In order to get rid of non-light line area and detect the light line length, the binarization and labelling processing are performed. Fig. 5 shows the result of binarization processing using threshold which was decided as mentioned above. In Fig. 5, although several non-light lines appear on the picture area, by labelling each separated area, the real running light line can be selected correctly. By calculate the length of the labelled light area, the vehicle speed can be derived.

\subsection{Calculate a real dimension of captured picture's width}

For calculating the real dimension, the relationship of position between the camera and the vehicle shown in Fig. 6 is needed. In Fig. 6, $h$ is an altitude of the camera from ground, $\theta$ is an elevator angle of the camera, $f$ is a focus distance of the camera, and $k$ is a width of an image sensor of the camera.

Using these parameters, the real dimension of captured picture width can be calculated by the following formula.

First, the Scaled width of image is calculated by following formula,

$$
x=2 L \tan \theta_{x}
$$

where

$$
\begin{aligned}
& L=\frac{h}{\cos \theta} \\
& \theta_{x}=2 \tan ^{-1}\left(\frac{k}{2 f}\right)
\end{aligned}
$$

Scaled distance per pixel is obtained from the following equation,

$$
p k=\frac{x}{m}
$$

where $m$ is the horizontal pixel number of the imaging sensor.

Finally, the vehicle speed $v$ is calculated by the following formula,

$$
v=p k \times d
$$

where $d$ is the moving light line length.

\section{Simulation}

In order to test the performance of this proposal, a simulation experiment was performed under the following conditions. The system setting information are $h=18[\mathrm{~m}]$, $\theta=45$ [degrees], $k=6.2[\mathrm{~mm}], f=4.3[\mathrm{~mm}]$.

The sample1 is Fig. 1. The sample1, the sample 2 of Fig. 8 , and the sample 3 of Fig. 11 is used as the test picture. Fig. 8 is the situation in which came out two line, and Fig. 11 is the situation in which blurred the line due to rain day. Fig., Fig. 9, and Fig. 12 shows the threshold-processed image from the sample1, the sample2, and the sample3. Moreover, Fig. 7, Fig. 10, and Fig. 13 show the extraction-processed image. The extracted line is expressed with green in Fig. 7, Fig. 10, and Fig. 13.

We also measure the vehicle speed by using a traditional stopwatch method for evaluation. The distance for stopwatch measuring is 30 meters. The comparing result is shown in Table 1. It seems that the proposed speed measuring approach gives a reasonable value, and it is thought can be used for a practical application.

TABLE I

\section{THE RESULT THAT CALCULATED THE} VEHICLE SPEED.

\begin{tabular}{|l|c|c|}
\hline & $\begin{array}{c}\text { The speed measured by } \\
\text { the system }[\mathrm{km} / \mathrm{h}]\end{array}$ & $\begin{array}{c}\text { The speed measured by } \\
\text { stopwatch }[\mathrm{km} / \mathrm{h}]\end{array}$ \\
\hline Sample1 & 41.6508 & 42.1 \\
\hline Sample2 & 41.65 & 45 \\
\hline Sample3 & 37.05 & 38.54 \\
\hline
\end{tabular}




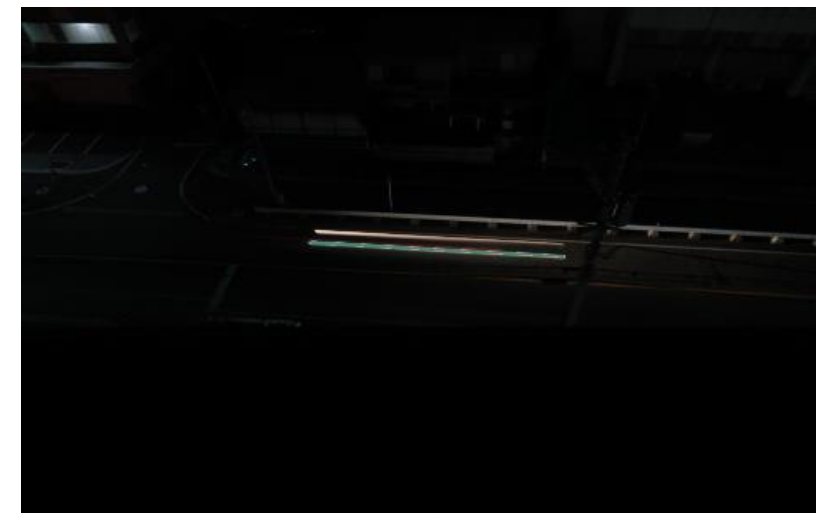

Fig. 7. Extraction of line sample1.

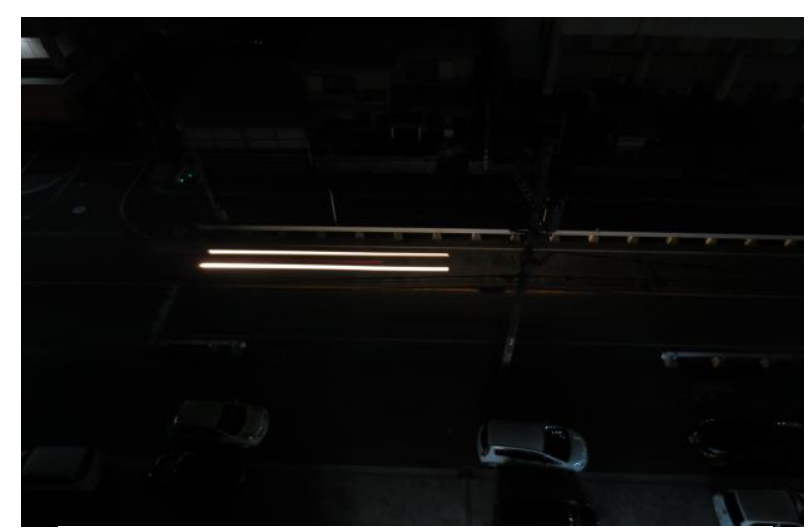

Fig. 8. Sample2.

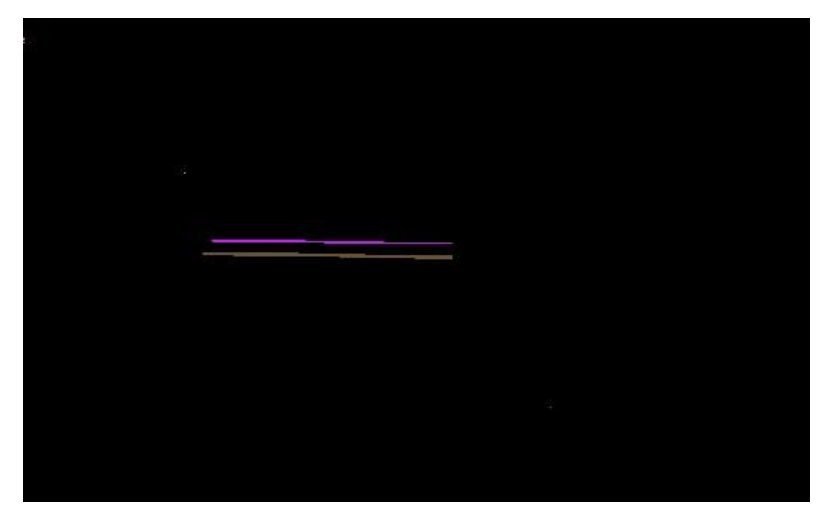

Fig. 9. Threshold-processed image of sample2.

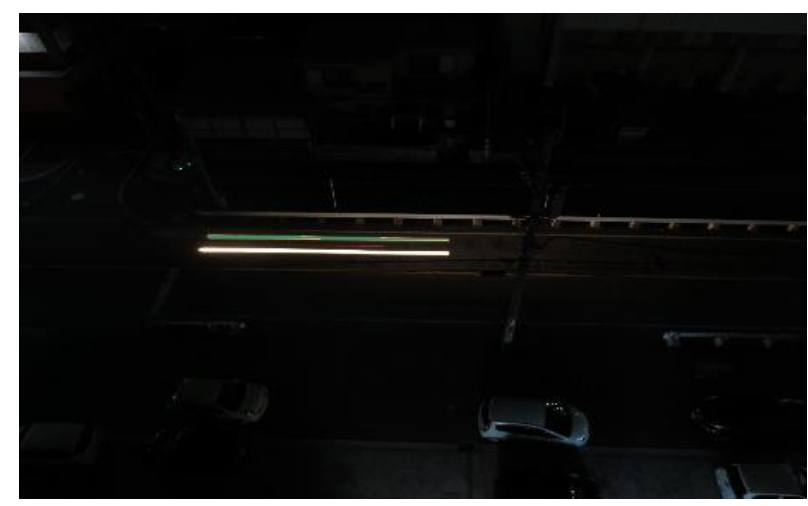

Fig. 10 Extraction of line sample2.

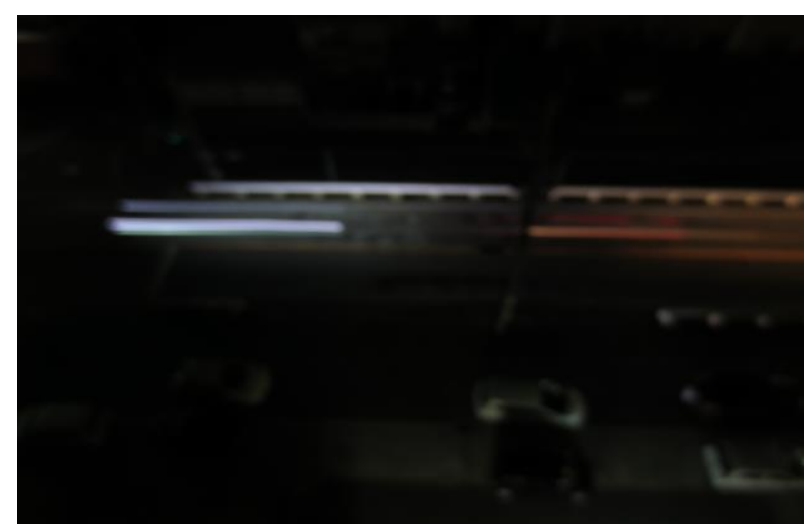

Fig. 11 Sample3.

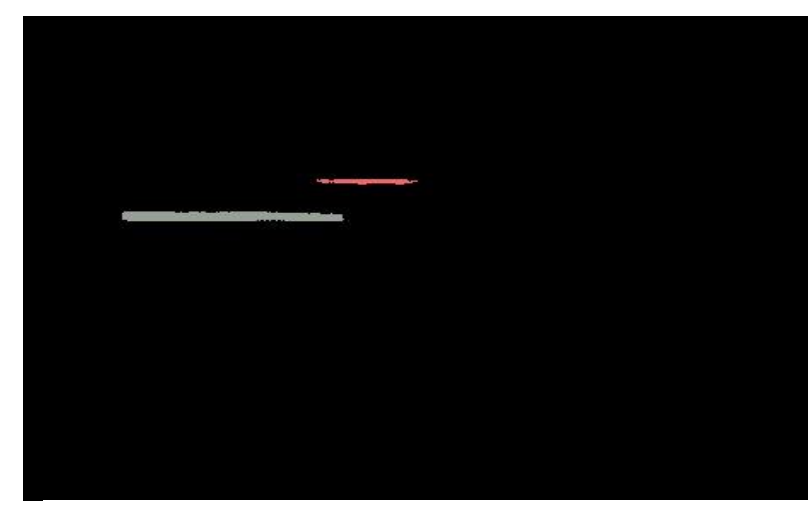

Fig. 12. Threshold-processed image of sample3.

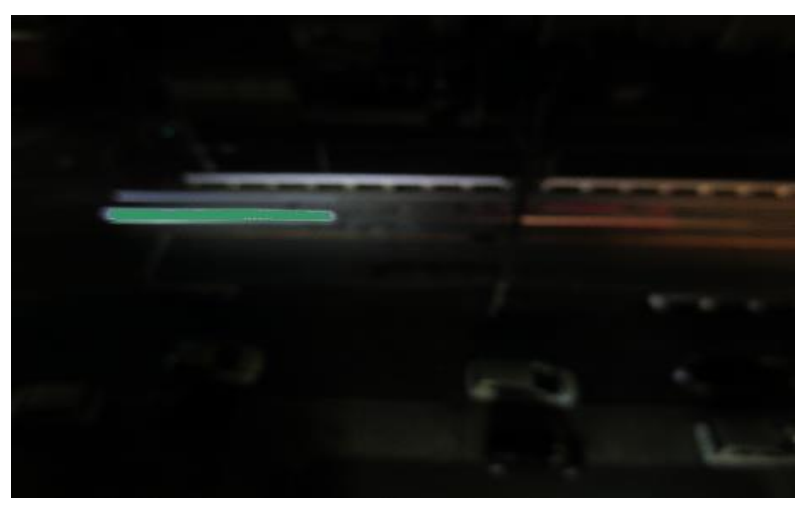

Fig. 13 Extraction of line sample3.

\section{Conclusions}

In this research, a new approach for vehicle speed measurement has been proposed. This method is especially developed for night environment. This system could yields a low cost and portable speed measuring equipment. This system has several setting terms. Because the line of light must be straight, this method cannot be used at the curve. Thereby, the setting terms of this system are to put the camera parallel to a straight road. However, this method 
controls the shutter speed. If the speed is set smaller than 1 second, the length of the light line will become short. Consequently, by changing the shutter speed, this system isn't affected by change of environment. We hope it could contribute to reducing the fatal car accident. But now the picture is taken manually, we still have a lot of future work like making an automatic photo taking function by installing an object detection sensor for shutter timing trigger. We also plan to make a system for the daytime, and to develop a small device which can measure the vehicle speed all time.

\section{References}

(1) Issues and review the status of speed limit http://www.e-stat.go.jp/SG1/estat/Pdfdl.do?sinfid=000 023620477 ,

accessed date: 2014/2/6

(2) Huff posted socity, http://www.huffingtonpost.jp/2013/09/21/ orbis_n_3966640.html, (In Janapese), accessed date: 2013/9/30

(3) Burt, Peter, and Edward Adelson. "The Laplacian pyramid as a compact image code." Communications, IEEE Transactions on 31.4, 532-540, 1983

(4) Tomasi, Carlo, and Roberto Manduchi. "Bilateral filtering for gray and color images." Computer Vision Sixth International Conference on. IEEE, 1998.

(5) $\mathrm{Hu}$, Weiming, et al. "A survey on visual surveillance of object motion and behaviors." Systems Man and Cybernetics Part C, Applications and Reviews, IEEE Transactions on 34.3, 334-352, 2004.

(6) Coifman, Benjamin, et al. "A real-time computer vision system for vehicle tracking and traffic surveillance." Transportation Research Part C, Emerging Technologies 6.4, 271-288, 1998.

(7) Cucchiara, Rita, Massimo Piccardi, and Paola Mello. "Image analysis and rule-based reasoning for a traffic monitoring system." Intelligent Transportation Systems, IEEE Transactions on 1.2, 119-130, 2000. 